\title{
Robust Stabilization of Discrete-Time Systems with Time-Varying Delay: An LMI Approach
}

\author{
Valter J. S. Leite ${ }^{1}$ and Márcio F. Miranda ${ }^{2}$ \\ ${ }^{1}$ Centro Federal de Educação Tecnológica de Minas Gerais (CEFET-MG), Campus Divinópolis, \\ Rua Monte Santo 319, Divinópolis 35502-036, MG, Brazil \\ ${ }^{2}$ Colégio Técnico (COLTEC), Universidade Federal de Minas Gerais (UFMG), Av. Antônio Carlos 6627, \\ Belo Horizonte 31270-901, MG, Brazil
}

Correspondence should be addressed to Valter J. S. Leite, valter@ieee.org

Received 29 June 2007; Revised 17 November 2007; Accepted 4 February 2008

Recommended by T. Runolfsson

\begin{abstract}
Sufficient linear matrix inequality (LMI) conditions to verify the robust stability and to design robust state feedback gains for the class of linear discrete-time systems with time-varying delay and polytopic uncertainties are presented. The conditions are obtained through parameter-dependent Lyapunov-Krasovskii functionals and use some extra variables, which yield less conservative LMI conditions. Both problems, robust stability analysis and robust synthesis, are formulated as convex problems where all system matrices can be affected by uncertainty. Some numerical examples are presented to illustrate the advantages of the proposed LMI conditions.
\end{abstract}

Copyright (C) 2008 V. J. S. Leite and M. F. Miranda. This is an open access article distributed under the Creative Commons Attribution License, which permits unrestricted use, distribution, and reproduction in any medium, provided the original work is properly cited.

\section{Introduction}

Time-delay systems have received a lot of attention from both academics and industrial engineers in the last decades. This can be verified by the great number of papers published in this area. See, for example, [1-5] and the references therein. It is worthwhile to mention that time delays affect both continuous and discrete-time systems and are presented in several systems like thermal processes, communication systems, internet dataflow, biological systems, regenerative chatter in metal cutting, and so on $[2,4]$. The presence of delay yields, in general, performance degradation and, eventually, leads the system to instability.

There are two main classes of robust stability analysis that have been investigated, namely, delay-dependent and delay-independent conditions. For a system whose stability does not depend on the time-delay value, the analysis performed through delay-dependent conditions can be very conservative. Also, delay-independent conditions cannot be obtained as 
a limit case of delay-dependent ones just by imposing the maximum delay value $d_{\max } \rightarrow+\infty$, leading to a gap between these two types of delay-stability conditions [4, page 146].

An important approach used in the last years to deal with delay in the states of the systems is the use of Lyapunov-Krasovskii functionals. This approach has been largely used to obtain convex conditions mainly for continuous-time systems subject to retarded states and for neutral systems [6]. On the other hand, discrete-time systems with state delay (DTSSD) have received little attention compared with its continuous-time counterpart. The main reason for this is that for precisely known discrete-time systems with constant time-delay, it is always possible to obtain an augmented system without delayed states [7]. This approach, however, does not seem to be suitable for time-varying delay, delay-independent stability characterization, and for robust-system stabilization. Despite the fact that the digital control has increased in importance for practical robust control applications in the last decades, there are few papers dealing with DTSSD. Besides this, the majority of the results available in the literature for DTSSD are formulated for norm-bounded uncertainty employing constant quadratic Lyapunov-Krasovskii functionals, which may lead to conservative results [8]. This approach, called quadratic stability, is the base of several results available in the literature as it can be seen in [9-12].

Recent results on DTSSD can be found in [13] where the real stability radii of timeinvariant state-delayed systems have been considered. In [14], there has been presented the descriptor approach to obtain stability and design conditions for discrete time-delay systems. See also [15] where some nonconvex conditions were proposed to the synthesis problem. In the context of systems with Markovian jumps, see $[3,16,17]$ and references therein. More recently, in $[18,19]$, robust stabilizing conditions have been proposed, but most of the results have been obtained through quadratic stability approach and the state feedback gain is designed directly from the Lyapunov-Krasovskii matrices. In [20], a dynamic controller is designed achieving a guaranteed cost control for norm-bounded DTSSD, but the design conditions are nonlinear. In [21], precisely known discrete-time systems with time-varying delays in the state are investigated and convex conditions are given to design a memoryless control law. The design conditions presented in [21] do not seem to be suitable to deal with polytopic systems since the matrices of the system multiply the Lyapunov-Krasovskii functional matrices. Observe that convex extensions of these results to treat polytopic type of uncertainty in the context of robust stability analysis and robust stabilization are not straightforward. Delay-independent conditions for robust stabilization of DTSSD have been considered in [22], where a parameter-dependent functional has been used, but only for time-invariant delays.

In this paper, new results for robust stability analysis as well as for robust stabilization of uncertain DTSSD with time-varying delay are proposed as convex conditions depending on the maximum and minimum values assumed by the delay, $\bar{d}$ and $\underline{d}$, respectively. A LyapunovKrasovskii functional similar to that employed in [21] is used. Differently from other results in the literature, both analysis and synthesis conditions are formulated as LMI feasibility problems that can be solved efficiently in polynomial time by specialized numerical algorithms. The main contribution of this paper is the improvement of the recently published results on DTSSD with time-varying delay given in [21]. Furthermore, the scope of the results of [21] is extended to deal with polytopic-type uncertainty affecting DTSSD and the possibility of design memory state feedback control gain. Parameter-dependent Lyapunov-Krasovskii functional used conjoint some extra matrices to achieve less conservative results. The approach proposed here 
does not introduce any additional dynamics and leads to a product separation between the matrices of the system and the matrices from the functional, allowing a convex formulation for the synthesis problem.

In Section 2 some definitions and the problem formulation are provided. Then, in Section 3, the main results are presented for both robust-stability analysis and for the synthesis of state feedback gains assuring the robust stability of the closed-loop system. The computational complexity of the proposed conditions and decentralized control design are discussed. In Section 4, some examples are given to illustrate the efficiency of the proposed conditions. In Section 5 some conclusions are presented.

Notation. The notation used here is quite standard. $\mathbb{R}$ is the set of real numbers and $\mathbb{N}$ is the set of natural numbers. $\mathbf{I}_{n}$ and $\mathbf{0}$ denote, respectively, the $n \times n$ identity matrix and the null matrix of appropriate dimensions. $M>\mathbf{0}(M<0)$ means that matrix $M$ is positive (negative) definite. $M^{\prime}$ is the transpose of $M$. The symbol $\star$ stands for symmetric blocks in the LMIs.

\section{Preliminaries and problem formulation}

Consider the following discrete-time system with a time-varying delay in the state:

$$
x_{k+1}=\tilde{A}(\alpha) x_{k}+\tilde{A}_{d}(\alpha) x_{k-d(k)}+\widetilde{B}(\alpha) u_{k}, \quad x_{k}=\phi(k), \quad k \in[-\bar{d}, 0],
$$

where $k$ is the sampling time, $x_{k} \in \mathbb{R}^{n}$ is the state vector, $u_{k} \in \mathbb{R}^{n}$ is the input control signal, $d(k)$ is the time-varying delay, where its time variation is bounded as

$$
\underline{d} \leq d(k) \leq \bar{d}, \quad(\underline{d}, \bar{d}) \in \mathbb{N} \times \mathbb{N}
$$

with $\underline{d}$ and $\bar{d}$ being the minimum and maximum delay values, respectively. $\left(\widetilde{A}(\alpha), \tilde{A}_{d}(\alpha)\right.$, $\widetilde{B}(\alpha)) \equiv\left(\widetilde{A}, \widetilde{A}_{d}, \widetilde{B}\right)(\alpha) \in \mathbb{R}^{n \times 2 n+\ell}$ are unknown constant matrices belonging to a polytope $\tilde{p}$,

$$
\begin{aligned}
\tilde{p} & \equiv\left\{\left(\widetilde{A}, \tilde{A}_{d}, \widetilde{B}\right)(\alpha):\left(\widetilde{A}, \tilde{A}_{d}, \widetilde{B}\right)(\alpha)=\sum_{i=1}^{N}\left(\widetilde{A}, \tilde{A}_{d}, \widetilde{B}\right)_{i} \alpha_{i}, \alpha \in \Omega\right\}, \\
\Omega & \equiv\left\{\alpha: \alpha \in \mathbb{R}^{N}, \sum_{i=1}^{N} \alpha_{i}=1, \alpha_{i} \geq 0\right\},
\end{aligned}
$$

where the vertices $\left(\widetilde{A}_{i}, \widetilde{A}_{d i}, \widetilde{B}_{i}\right) \equiv\left(\widetilde{A}, \widetilde{A}_{d}, \widetilde{B}\right)_{i}$ are precisely known. In special, observe that if $\underline{d}=\bar{d}$, then the delay is uncertain, belonging to $[0, \bar{d}]$, but it is time invariant. Note that if $\bar{d}(k)=0$, then $(2.1)$ is rewritten as $x_{k+1}=\left(\widetilde{A}(\alpha)+\widetilde{A}_{d}(\alpha)\right) x_{k}+\widetilde{B}(\alpha) u_{k}$. Also define

$$
\beta=\bar{d}-\underline{d}+1 \text {. }
$$

In this paper, the following control law is considered:

$$
u(k)=K x(k)+K_{d} x(k-d(k)),
$$

where $\left(K, K_{d}\right) \in \mathbb{R}^{\ell \times 2 n}$ are the robust state feedback gains that assure the robust stability of the closed-loop system, that is, the stability of (2.1)-(2.4) with (2.6) is assured for all $\alpha \in \Omega$. Therefore, this uncertain closed-loop system is given by

$$
x_{k+1}=A(\alpha) x_{k}+A_{d}(\alpha) x_{k-d(k)}
$$


with

$$
A(\alpha) \equiv \widetilde{A}(\alpha)+\widetilde{B}(\alpha) K, \quad A_{d}(\alpha) \equiv \tilde{A}_{d}(\alpha)+\widetilde{B}(\alpha) K_{d}
$$

where $\left(A, A_{d}\right)(\alpha) \in \mathcal{D}$ with

$$
p \equiv\left\{\left(A, A_{d}\right)(\alpha):\left(A, A_{d}\right)(\alpha)=\sum_{i=1}^{N}\left(A, A_{d}\right)_{i} \alpha_{i}, \alpha \in \Omega\right\}
$$

It is worth to mention that if the delay $d(k)$ is not known at each sample time $k$, then it is enough to make $K_{d}=0$ in (2.6). If $d(k)$ is known at each sample time $k$, then the possibility of using $K$ and $K_{d}$ may improve the performance of the closed-loop system (2.7).

The objective of this paper is to give convex conditions solving the following problems.

Problem 1. Given $\underline{d}$ and $\bar{d}$ subject to (2.2), determine if the uncertain DTSSD given in (2.7) is robustly stable.

Problem 2. Find a pair of gains $\left(K, K_{d}\right)$ such that the system $(2.1)-(2.4)$ controlled by $(2.6)$ is robustly stable.

Remark 2.1. Generally speaking, in the cases where the time-delay depends on a physical parameter (such as velocity of a transport belt, the stem position of a valve, etc.) it may be possible to determine the delay value at each sample-time. As a special case, consider the regenerative chatter in metal cutting. In this process a cylindrical workpiece has an angular velocity $\omega$ while a machine tool (lathe) translates along the axis of this workpiece. For details, see [2, page 2]. In this case the delay depends on the velocity $\omega$ and thus, if this angular velocity can be measured, the delay could be determined at each instant. Note, however, that a detailed study on physical application is not the focus of the paper.

\section{Main results}

First, sufficient LMI conditions to solve Problem 1 are given. The approach used here does not introduce any dynamics and leads to a product separation between the matrices of the system and those from the Lyapunov-Krasovskii functional. Then, these conditions are exploited to provide some convex synthesis results. The following theorems provide some LMI conditions depending on the values $\bar{d}$ and $\underline{d}$ to determine the robust stability of (2.7) or to design robust state feedback gains $K$ and $K_{d}$ that assure the robust closed-loop stability.

\subsection{Robust stability analysis}

Theorem 3.1. System (2.7) subject to (2.2), (2.4), and (2.9) is robustly stable if there exist symmetric matrices $\mathbf{0}<P(\alpha) \in \mathbb{R}^{n \times n}$ and $\mathbf{0}<Q(\alpha) \in \mathbb{R}^{n \times n}$, such that one of the following equivalent conditions is verified:

(a)

$$
\Gamma(\alpha) \equiv\left[\begin{array}{cc}
A(\alpha)^{\prime} P(\alpha) A(\alpha)+\beta Q(\alpha)-P(\alpha) & A(\alpha)^{\prime} P(\alpha) A_{d}(\alpha) \\
\star & A_{d}(\alpha)^{\prime} P(\alpha) A_{d}(\alpha)-Q(\alpha)
\end{array}\right]<0
$$


(b) there exist parameter-dependent matrices $F(\alpha) \in \mathbb{R}^{n \times n}, G(\alpha) \in \mathbb{R}^{n \times n}$, and $H(\alpha) \in \mathbb{R}^{n \times n}$, such that

$$
\mathcal{M}(\alpha) \equiv\left[\begin{array}{ccc}
P(\alpha)+F(\alpha)^{\prime}+F(\alpha) & G(\alpha)^{\prime}-F(\alpha) A(\alpha) & H(\alpha)^{\prime}-F(\alpha) A_{d}(\alpha) \\
\star & \vartheta_{22}(\alpha) & -A(\alpha)^{\prime} H(\alpha)^{\prime}-G(\alpha) A_{d}(\alpha) \\
\star & \star & \vartheta_{33}(\alpha)
\end{array}\right]<\mathbf{0},
$$

where $\vartheta_{22}(\alpha)$ denotes $-A(\alpha)^{\prime} G(\alpha)^{\prime}-G(\alpha) A(\alpha)+\beta Q(\alpha)-P(\alpha)$ and $\vartheta_{33}(\alpha)$ denotes $-(Q(\alpha)+$ $\left.H(\alpha) A_{d}(\alpha)+A_{d}(\alpha)^{\prime} H(\alpha)^{\prime}\right)$ with $\beta$ given by (2.5). In this case, the functional

$$
V(\alpha, k)=\sum_{v=1}^{3} V_{v}(\alpha, k)>0,
$$

with

$$
\begin{aligned}
& V_{1}(\alpha, k)=x_{k}^{\prime} P(\alpha) x_{k}, \\
& V_{2}(\alpha, k)=\sum_{j=k-d(k)}^{k-1} x_{j}^{\prime} Q(\alpha) x_{j}, \\
& V_{3}(\alpha, k)=\sum_{\ell=2-\bar{d}}^{1-d} \sum_{j=k+\ell-1}^{k-1} x_{j}^{\prime} Q(\alpha) x_{j},
\end{aligned}
$$

is such that

$$
\Delta V(\alpha, k)<0, \quad \forall\left[x(k)^{\prime} x(k-d(k))^{\prime}\right]^{\prime} \neq 0
$$

and is called a Lyapunov-Krasovskii functional, assuring the robust stability of (2.7).

Proof. The positivity of the functional (3.3) is assured with the hypothesis of $P(\alpha)=P(\alpha)^{\prime}>0$, $Q(\alpha)=Q(\alpha)^{\prime}>0$. For (3.3) is a Lyapunov-Krasovskii functional, besides its positivity, it is necessary to verify (3.5) for all $\alpha \in \Omega$. From hereafter, the $\alpha$ dependency is omitted in the expressions $V_{v}(k), v=1, \ldots, 3$, for simplicity of the notation. To calculate (3.5), consider

$$
\begin{aligned}
& \Delta V_{1}(k)=x_{k+1}^{\prime} P(\alpha) x_{k+1}-x_{k}^{\prime} P(\alpha) x_{k}, \\
& \Delta V_{2}(k)=x_{k}^{\prime} Q(\alpha) x_{k}-x_{k-d(k)}^{\prime} Q(\alpha) x_{k-d(k)}+\sum_{i=k+1-d(k+1)}^{k-1} x_{i}^{\prime} Q(\alpha) x_{i}-\sum_{i=k+1-d(k)}^{k-1} x_{i}^{\prime} Q(\alpha) x_{i}, \\
& \Delta V_{3}(k)=(\bar{d}-\underline{d}) x_{k}^{\prime} Q(\alpha) x_{k}-\sum_{i=k+1-\bar{d}}^{k-d} x_{i}^{\prime} Q(\alpha) x_{i} .
\end{aligned}
$$

Observe that the third term in (3.7) can be rewritten as

$$
\begin{aligned}
\Xi_{k} \equiv \sum_{i=k+1-d(k+1)}^{k-1} x_{i}^{\prime} Q(\alpha) x_{i} & =\sum_{i=k+1-\underline{d}}^{k-1} x_{i}^{\prime} Q(\alpha) x_{i}+\sum_{i=k+1-d(k+1)}^{k-d} x_{i}^{\prime} Q(\alpha) x_{i} \\
& \leq \sum_{i=k+1-d(k)}^{k-1} x_{i}^{\prime} Q(\alpha) x_{i}+\sum_{i=k+1-\bar{d}}^{k-d} x_{i}^{\prime} Q(\alpha) x_{i} .
\end{aligned}
$$


Using (3.9) in (3.7), one gets

$$
\Delta V_{2}(k) \leq x_{k}^{\prime} Q(\alpha) x_{k}-x_{k-d(k)}^{\prime} Q(\alpha) x_{k-d(k)}+\sum_{i=k+1-\bar{d}}^{k-d} x_{i}^{\prime} Q(\alpha) x_{i} .
$$

So, taking into account (3.6), (3.8), and (3.10) the following upper bound for (3.5) can be obtained:

$$
\Delta V(k) \leq x_{k+1}^{\prime} P(\alpha) x_{k+1}+x_{k}^{\prime}[\beta Q(\alpha)-P(\alpha)] x_{k}-x_{k-d(k)}^{\prime} Q(\alpha) x_{k-d(k)}<0 .
$$

Replacing $x_{k+1}$ in (3.11) by the right-hand side of (2.7) one gets (3.1). The equivalence between (3.1) and (3.2) can be established as follows. First, note that (3.1) can be rewritten as

$$
\left[\begin{array}{c}
A(\alpha)^{\prime} P(\alpha) \\
A_{d}(\alpha)^{\prime} P(\alpha)
\end{array}\right] P(\alpha)^{-1}\left[P(\alpha) A(\alpha) P(\alpha) A_{d}(\alpha)\right]-\left[\begin{array}{cc}
P(\alpha)-\beta Q(\alpha) & 0 \\
0 & Q(\alpha)
\end{array}\right]<0
$$

which by Schur complement is equivalent to

$$
\left[\begin{array}{ccc}
-P(\alpha) & P(\alpha) A(\alpha) & P(\alpha) A_{d}(\alpha) \\
\star & \beta Q(\alpha)-P(\alpha) & 0 \\
\star & \star & -Q(\alpha)
\end{array}\right]<0
$$

Therefore, the equivalence between (a) and (b) is the same as that between (3.2) and (3.13). So, if (3.13) is verified, then (3.2) is true for $F(\alpha)=F(\alpha)^{\prime}=-P(\alpha), G(\alpha)=H(\alpha)=0$. On the other hand, if (3.2) is verified, then $\Gamma(\alpha)=T(\alpha)^{\prime} \mathcal{M}(\alpha) T(\alpha)$ with

$$
T(\alpha)=\left[\begin{array}{cc}
A(\alpha) & A_{d}(\alpha) \\
\mathbf{I}_{n} & \mathbf{0} \\
\mathbf{0} & \mathbf{I}_{n}
\end{array}\right]
$$

completing the proof.

Note that (3.7) keeps a relation with Finsler's lemma where the slack variables $F(\alpha)$ and $G(\alpha)$ depend on the uncertain parameter $\alpha$. The conditions presented in Theorem 3.1 are of infinite dimension if $\alpha$ belongs to a continuous domain. These conditions can be numerically treated by using different approaches such as those presented in [23,24], where it is possible to consider the products of matrices depending on $\alpha$ by means of LMI relaxations. In case of $\alpha$ belonging to a discrete, countable, and finite domain, the conditions of Theorem 3.1 state a finite set of LMIs defined at each value of $\alpha$. In this paper, the structure of matrices $P(\alpha)$ and $Q(\alpha)$ is supposed to be linear in $\alpha$ [25]:

$$
P(\alpha)=\sum_{i=1}^{N} \alpha_{i} P_{i}, \quad Q(\alpha)=\sum_{i=1}^{N} \alpha_{i} Q_{i}
$$

and $\alpha \in \Omega$. The extra matrices are chosen to be fixed $F(\alpha)=F, G(\alpha)=G$, and $H(\alpha)=H$. Although other structures may lead to a less conservative condition for Problem 1, no improvement is expected for Problem 2 as it deals with constant state feedback gains $K$ and $K_{d}$. Also observe that, since the conditions of Theorem 3.1 depend on the size of delay variation, $\bar{d}-\underline{d}$, and not on the delay value itself, these conditions are delay-independent conditions. 
Theorem 3.2. System (2.7) subject to (2.2), (2.4), and (2.9) is robustly stable if there exist symmetric matrices $0<P_{i} \in \mathbb{R}^{n \times n}$ and $\mathbf{0}<Q_{i} \in \mathbb{R}^{n \times n}, i=1, \ldots, N$, such that

$$
\mathcal{M}_{i} \equiv\left[\begin{array}{ccc}
P_{i}+F^{\prime}+F & G^{\prime}-F A_{i} & H^{\prime}-F A_{d i} \\
\star & \beta Q_{i}-P_{i}-A_{i}^{\prime} G^{\prime}-G A_{i} & -A_{i}^{\prime} H^{\prime}-G A_{d i} \\
\star & \star & -\left(Q_{i}+H A_{d i}+A_{d i}^{\prime} H^{\prime}\right)
\end{array}\right]<0, \quad i=1, \ldots, N,
$$

are verified with $\beta$ given by (2.5). Beside this, (3.3) with (3.4) and (3.15) is a Lyapunov-Krasovskii functional for (2.7).

Proof. Observe that $\mathcal{M}(\alpha)$ can be obtained by multiplying (3.16) by $\alpha_{i}$ and summing it up, that is, $\mathcal{M}(\alpha)=\sum_{i=1}^{N} \alpha_{i} \mathcal{M}_{i}, \alpha \in \Omega$.

It is worth to mention that the parameter-dependent structure, imposed to $P(\alpha)$ and $Q(\alpha)$, (3.15), cannot be directly used in the conditions presented in (3.1) and (3.13). This limitation is due to the products between the system matrices and the Lyapunov-Krasovskii candidate matrices.

The approach based on quadratic stability can be recovered from (3.1), (3.2), (3.13) (or (3.16)). In this case, it is sufficient to impose $P(\alpha)=P, Q(\alpha)=Q\left(P_{i}=P, Q_{i}=Q, i=1, \ldots, N\right)$ and replace $A(\alpha)$ and $A_{d}(\alpha)$ by $A_{i}$ and $A_{d i}$, respectively. Then it is necessary to test those conditions for $i=1, \ldots, N$. Note that all the quadratic stability conditions obtained as described above are equivalent and the difference between them is just the computational burden necessary to solve each one.

Observe that conditions presented in Theorems 3.1 and 3.2 can be used to test the robust stability of both systems $\left(A(\alpha), A_{d}(\alpha)\right)$ and $\left(A(\alpha)^{\prime}, A_{d}(\alpha)^{\prime}\right)$, since their eigenvalues are the same.

\subsection{Robust feedback design}

The stability analysis conditions presented in Theorem 3.2 can be used to obtain a convex condition for robust synthesis of the gains $K$ and $K_{d}$ such that the control law (2.6) applied in (2.1) results in a robust stable closed-loop system, and, therefore, resulting in a solution to Problem 2.

Theorem 3.3. If there exist symmetric matrices $0<P_{i} \in \mathbb{R}^{n \times n}, \mathbf{0}<Q_{i} \in \mathbb{R}^{n \times n}, i=1, \ldots, N$, matrices $F \in \mathbb{R}^{n \times n}, W \in \mathbb{R}^{n \times \ell}$, and $W_{d} \in \mathbb{R}^{n \times \ell}$, such that the following LMIs

$$
\left[\begin{array}{ccc}
P_{i}+F+F^{\prime} & -\left(F \tilde{A}_{i}^{\prime}+W \widetilde{B}_{i}^{\prime}\right) & -\left(F \widetilde{A}_{d i}^{\prime}+W_{d} \widetilde{B}_{i}^{\prime}\right) \\
\star & \beta Q_{i}-P_{i} & 0 \\
\star & \star & -Q_{i}
\end{array}\right]<0, \quad i=1, \ldots, N
$$

are verified with $\beta$ given by (2.5), then system given by (2.1)-(2.4) is robustly stabilizable with (2.6), where the static feedback gains are given by

$$
K=W^{\prime}\left(F^{\prime}\right)^{-1}, \quad K_{d}=W_{d}^{\prime}\left(F^{\prime}\right)^{-1}
$$

yielding a convex solution to Problem 2. Beside this, (3.3)-(3.4) is a Lyapunov-Krasovskii functional that guarantees the robust stability of the resulting closed-loop system with $P(\alpha)$ and $Q(\alpha)$ given in (3.15). 
Proof. Condition (3.17) is obtained from (3.16) by choosing $G=H=\mathbf{0}$, replacing $A_{i}$ and $A_{d i}$ by $\left(\widetilde{A}_{i}+\widetilde{B}_{i} K\right)^{\prime}$ and $\left(\widetilde{A}_{d i}+\widetilde{B}_{i} K_{d}\right)^{\prime}$, respectively, and making the change of variables $F K^{\prime}=W$ and $F K_{d}^{\prime}=W_{d}$.

Note that conditions presented in Theorem 3.3 encompass quadratic stability, since it is always possible to choose $P_{i}=P$ and $Q_{i}=Q, i=1, \ldots, N$. Also observe that if $d(k)$ is not available at each sample, and therefore $x(k-d(k))$ cannot be used in the feedback, then it is enough to choose $W_{d}=0$ leading to a control law given by $u(k)=K x(k)$. Another relevant note is that Theorem 3.3 presents some LMI conditions for the synthesis of state feedback gains, differently from other approaches found in the literature where nonconvex techniques are employed.

\section{Decentralized control}

The results of Theorem 3.3 can be used to deal with decentralized control. This can be done by imposing a diagonal structure on $F=F_{D}=\operatorname{block}$-diag $\left\{F_{1}, \ldots, F_{\kappa}\right\}, W=W_{D}=$ block-diag $\left\{W_{1}, \ldots, W_{\kappa}\right\}$, and $W_{d}=W_{d D}=$ block-diag $\left\{W_{d 1}, \ldots, W_{d \kappa}\right\}, \kappa$ being the number of subsystems. In this case, robust block-diagonal state feedback gains given by $K_{D}=W_{D}^{\prime}\left(F_{D}^{\prime}\right)^{-1}$ and $K_{d D}=W_{d D}^{\prime}\left(F_{D}^{\prime}\right)^{-1}$ can be obtained. Beside this, no structure is imposed to the matrices of the Lyapunov-Krasovskii functional, $P(\alpha)$ and $Q(\alpha)$, which may lead to less conservative results.

\section{Computational complexity}

The computational complexity of the conditions presented in this paper can be determined by the number of scalar variables, $\mathcal{K}$, and the number of rows, $\mathcal{R}$, involved in the optimization problems. Theorem 3.2 presents $\mathcal{K}_{T 2}=n[3 n+N(n+1)]$ scalar variables and $\mathcal{R}_{T 2}=5 N n$ LMI rows and in Theorem 3.3 it is found that $\mathcal{K}_{T 3}=n[2 \ell+n+N(n+1)]$ and $\mathcal{R}_{T 3}=3 N n$. In the case of using LMI control toolbox [26], the computational complexity is $\mathcal{O}\left(\mathcal{K}^{3} \mathcal{R}\right)$ and using the solver SeDuMi [27] the computational complexity is $\mathcal{O}\left(\mathcal{K}^{2} R^{2.5}+R^{3.5}\right)$.

\section{Numerical examples}

Example 4.1. This example shows that the condition presented by Theorem 3.2 can be less conservative than other conditions available in the literature. Consider the DTSSD given by (2.7), where

$$
A_{1}=\left[\begin{array}{cc}
0.6 & 0 \\
0.35 & 0.7
\end{array}\right], \quad A_{d 1}=\left[\begin{array}{cc}
0.1 & 0 \\
0.2 & 0.1
\end{array}\right] .
$$

This system has been investigated in [18] and its stability has been assured for $2 \leq d(k) \leq 13$ by using a Lyapunov-Krasovskii with 5 terms instead of the 3 terms presented here (see (3.3)(3.4)). Using Theorem 3.2 the same delay interval is obtained, but with a lower-computational complexity. Using [21, Theorem 3.1] that employs a Lyapunov-Krasovskii functional similar to that used here, it is possible to verify the stability of this system but with a narrower delay interval: $2 \leq d(k) \leq 10$.

Consider now that this system is affected by an uncertain parameter such that it can be described by a polytope (2.3) with $A_{1}$ and $A_{d 1}$ given above and $A_{2}=1.1 A_{1}$ and $A_{d 2}=1.1 A_{d 1}$. 
In this case, the conditions of $[18,21]$ are no longer applicable, since the system is uncertain. Using Theorem 3.2, it is possible to assure the robust stability of this system for $|d(k+1)-d(k)| \leq$ 3. Thus, a more flexible analysis condition is provided by Theorem 3.2.

Example 4.2. Consider the discrete-time system with delayed states which is described by (2.1), where the system matrices are given by

$$
\tilde{A}=\left[\begin{array}{cc}
0.00 & 1.00 \\
-2.00 & -3.00
\end{array}\right], \quad \tilde{A}_{d}=\left[\begin{array}{ll}
0.01 & 0.10 \\
0.00 & 0.10
\end{array}\right], \quad \tilde{B}=\left[\begin{array}{l}
0.00 \\
1.00
\end{array}\right] .
$$

This system has been investigated for $\underline{d}=1 \leq d(k) \leq 10=\bar{d}$ in [21] where a state feedback gain $K=[2.0005,2.9051]$ has been obtained by means of application of its Theorem 4.1 that has $\mathcal{K}[21]=19$ scalar variables and $R[21]=16$ rows. On the other hand, the conditions of Theorem 3.3 have $\mathcal{K}_{T 3}=12$ scalar variables and $\mathcal{R}_{T 3}=6$ rows. Thus, by using LMI control toolbox [26], the condition proposed in [21] is more complex than that in Theorem 3.3. Conditions of Theorem 3.3 yield

$$
Q=\left[\begin{array}{ll}
0.1558 & 0.0003 \\
0.0003 & 0.0209
\end{array}\right], \quad P=\left[\begin{array}{cc}
2.3902 & -0.0026 \\
-0.0026 & 0.4315
\end{array}\right], \quad F=\left[\begin{array}{cc}
-1.5441 & 0.0047 \\
0.0005 & -0.4866
\end{array}\right]
$$

and $K=[2.0000,2.9929]$, which is close to the gain obtained by [21] with a lower computational cost. Therefore, this example illustrates that the conditions proposed here are numerically more efficient than those proposed in [21].

Example 4.3. Consider the system investigated in Example 4.2 where some uncertainties have been added as follows:

$$
\widetilde{A}(\rho)=(1+\rho) \tilde{A}, \quad \widetilde{A}_{d}(\theta)=(1+\theta) \tilde{A}_{d}, \quad \widetilde{B}(\eta)=(1+\eta) \widetilde{B}
$$

with $|\rho| \leq 0.07,|\theta| \leq 0.1$, and $|\eta| \leq 0.1$. These parameters lead to a polytope with 8 vertices determined by the combination of the extreme values of $\rho, \theta$, and $\eta$. Keeping the same delay variation interval considered in Example 4.2, that is, $\underline{d}=1$ and $\bar{d}=10$, the conditions of Theorem 3.3 are used to stabilize this uncertain system resulting in

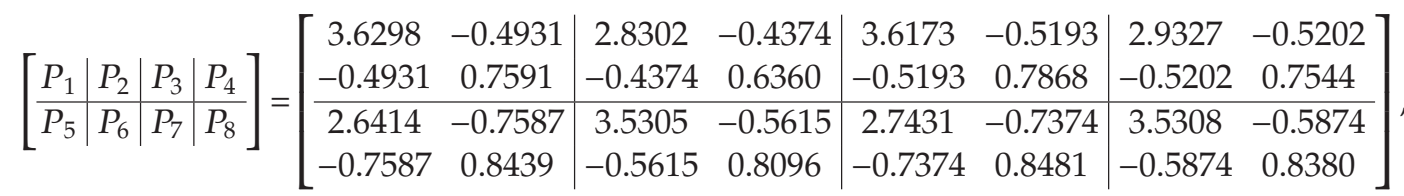

$$
\begin{aligned}
& {\left[\begin{array}{c|c|c|c}
Q_{1} & Q_{2} & Q_{3} & Q_{4} \\
\hline Q_{5} & Q_{6} & Q_{7} & Q_{8}
\end{array}\right]=\left[\begin{array}{cc|cc|cc|cc}
0.1976 & -0.0171 & 0.1292 & -0.0306 & 0.2015 & -0.0212 & 0.1515 & -0.0385 \\
-0.0171 & 0.0538 & -0.0306 & 0.0326 & -0.0212 & 0.0550 & -0.0385 & 0.0396 \\
\hline 0.0382 & 0.0138 & 0.2055 & -0.0194 & 0.0847 & -0.0013 & 0.2102 & -0.0241 \\
0.0138 & 0.0207 & -0.0194 & 0.0527 & -0.0013 & 0.0237 & -0.0241 & 0.0543
\end{array}\right],} \\
& F=\left[\begin{array}{cc}
-2.4178 & 0.5476 \\
0.5056 & -0.7569
\end{array}\right] \text {, } \\
& K=\left[\begin{array}{ll}
1.9670 & 2.7170
\end{array}\right] \text {. }
\end{aligned}
$$




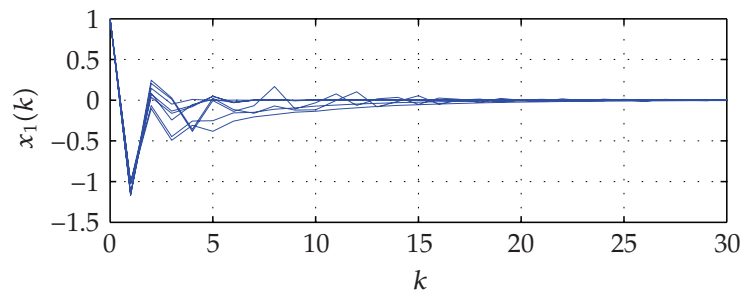

(a)

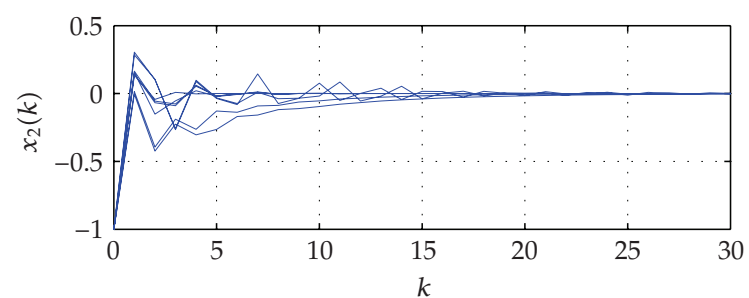

(b)

Figure 1: The behavior of the states $x_{1}(k)$ (a) and $x_{2}(k)$, with $(\mathrm{b}) 1 \leq d(k) \leq 10$ (see Figure $2(\mathrm{~b})$ ), $K=$ [109670, 2.7170].

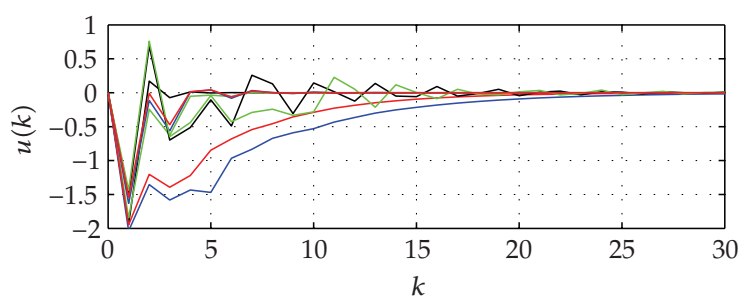

(a)

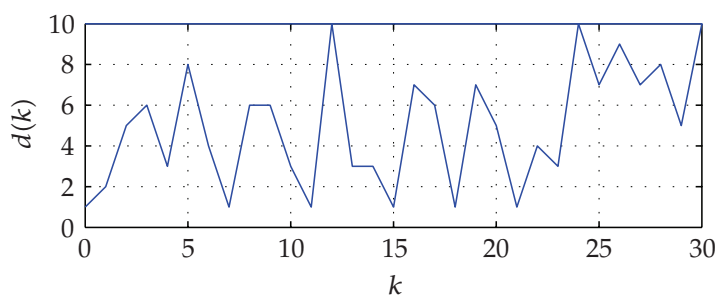

(b)

Figure 2: Control signal (a) and time-varying delay (b).

The behavior of the states of the closed-loop response of this uncertain discrete-time system with time-varying delay is shown in Figure 1. It has been simulated that the time response of this system at each vertex of the polytope was used in the controller design. The respective control signals are shown in Figure 2. The initial conditions have been chosen as 
$x(k)=[1,-1], k=-\bar{d}, \ldots, 0$, and the value of the delay, $d(k)$, has been varied randomly as shown in Figure 2(b). In Figure 1, the stability of the uncertain closed-loop system is illustrated, assured by the state feedback calculated by means of Theorem 3.3, for $1 \leq d(k) \leq 10$. The control effort is shown in Figure 2(a). It is worth to mention that the results presented in [21] cannot be directly used with polytopic uncertain systems and cannot be applied in this case. This example shows the efficacy of the conditions proposed here applied to uncertain discrete-time delayed systems.

Example 4.4. In this example, it is shown how conditions of Theorem 3.3 can be used to design robust decentralized state feedback gains. Consider the system (2.1) with 4 states and 2 vertex matrices given by

$$
\begin{aligned}
{\left[\widetilde{A}_{1} \mid \widetilde{A}_{2}\right] } & =\left[\begin{array}{cccc|cccc}
0.90 & 0.00 & -0.08 & 0.03 & 0.90 & 0.00 & -0.08 & 0.03 \\
0.00 & 0.70 & 0.05 & -0.03 & 0.00 & 0.90 & 0.05 & -0.03 \\
-0.08 & 0.05 & -0.29 & 1.00 & -0.04 & 0.03 & -0.10 & 0.00 \\
0.03 & -0.03 & 0.00 & 0.95 & 0.01 & -0.01 & 1.00 & -0.20
\end{array}\right], \\
{\left[\widetilde{A}_{d 1} \mid \widetilde{A}_{d 2}\right] } & =\left[\begin{array}{cccc|cccc}
-0.10 & 0.00 & -0.10 & 0.03 & -0.10 & 0.10 & -0.10 & 0.06 \\
-0.10 & -0.10 & 0.06 & -0.03 & -0.10 & -0.10 & 0.03 & -0.03 \\
-0.10 & 0.06 & 0.01 & 0.01 & -0.10 & 0.06 & 0.01 & 0.00 \\
0.03 & -0.03 & 0.00 & 0.02 & 0.03 & -0.03 & 0.01 & 0.02
\end{array}\right], \\
{\left[\widetilde{B}_{1} \mid \widetilde{B}_{2}\right] } & =\left[\begin{array}{cccc|cccc}
1 & 0 & 0 & 0 & 0 & 0 & 0 & 0 \\
0 & 1 & 0 & 0 & 1 & 0 & 0 & 0 \\
0 & 0 & 1 & 0 & 0 & 0 & 1 & 1 \\
0 & 0 & 0 & 0 & 0 & 0 & 0.1 & 0
\end{array}\right] .
\end{aligned}
$$

By imposing a block diagonal structure on $F$ and $W$ given by $\{2,2\}$ and choosing $W_{d}=\mathbf{0}$, it is possible to apply the conditions of Theorem 3.3 getting

$$
K=\left[\begin{array}{cc}
K_{1} & 0 \\
\mathbf{0} & K_{2}
\end{array}\right] \quad \text { with } K_{1}=\left[\begin{array}{cc}
0.4873 & -0.8785 \\
1.6425 & -1.7437
\end{array}\right], K_{2}=\left[\begin{array}{cc}
0.2587 & -0.6089 \\
0.2299 & 0.4833
\end{array}\right]
$$

for $\underline{d}=1$ and $\bar{d}=4$. Thus, this example shows how the proposed synthesis conditions can be used to design robust decentralized state feedback control gains without imposing any structure on Lyapunov-Krasovskii functional matrices, yielding full matrices $P_{i}$ and $Q_{i}, i=1,2$.

Example 4.5. This last example is presented to illustrate how the proposed conditions can be used within time-varying systems, that is, time-varying delay systems with matrices $\widetilde{A}, \widetilde{A}_{d}$, and $\widetilde{B}$ depending on $\alpha(t)$. Consider the uncertain discrete-time system with time-varying delay 


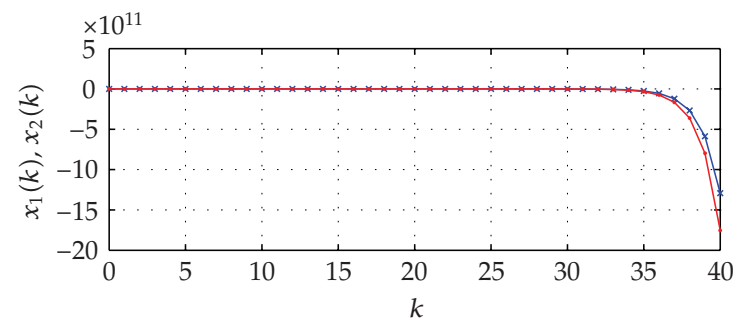

Figure 3: The behavior of the states $x_{1}(k)(\times)$ and $x_{2}(k)(\cdot)$ for the unforced system with $1 \leq d(k) \leq 2$ and $\alpha_{1}=0.4$.

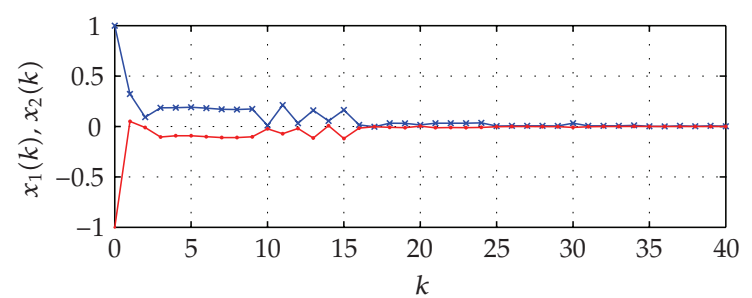

(a)

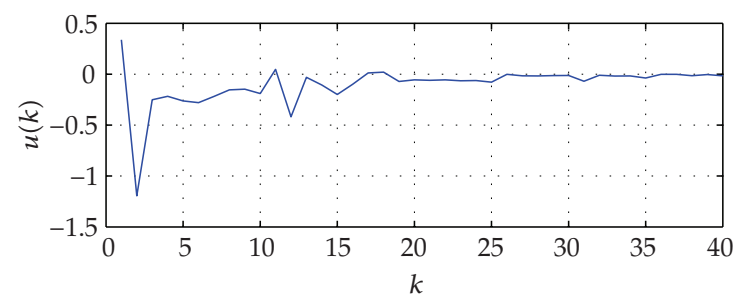

(b)

Figure 4: The behavior of the states $x_{1}(k)(\times)$ and $x_{2}(k)(\cdot)$ on (a) and the control signal on (b).

described by a polytope of matrices with vertices given by

$$
\begin{aligned}
{\left[\tilde{A}_{1} \mid \tilde{A}_{2}\right] } & =\left[\begin{array}{ll|ll}
1.33 & 1.26 & 0.37 & 0.74 \\
1.49 & 1.46 & 0.91 & 1.14
\end{array}\right], \\
{\left[\tilde{A}_{d 1} \mid \tilde{A}_{d 2}\right] } & =\left[\begin{array}{ll|cc}
0.20 & 0.06 & 0.16 & -0.06 \\
0.01 & 0.14 & -0.01 & 0.06
\end{array}\right], \\
{\left[\widetilde{B}_{1} \mid \widetilde{B}_{2}\right] } & =\left[\begin{array}{l|l|l}
0.39 & 0.11 \\
0.48 & 0.32
\end{array}\right] .
\end{aligned}
$$

The open-loop system is not robustly stable as can be noticed by the eigenvalues of $A_{1}$, given by 0.0233 and 2.7667 , and the eigenvalues of $A_{2}$, given by -0.1514 and 1.6614 . The unforced system is simulated for an initial state given by $x_{k}=[1,-1]^{\prime}, k \in[-2,0]$ and a constant $\alpha_{1}=0.4$, that is, $\widetilde{A}(\alpha)=0.4 \widetilde{A}_{1}+0.6 \widetilde{A}_{2}, \widetilde{A}_{d}(\alpha)=0.4 \widetilde{A}_{d 1}+0.6 \widetilde{A}_{d 2}$, and $\widetilde{B}(\alpha)=0.4 \widetilde{B}_{1}+0.6 \widetilde{B}_{2}$. The unstable behavior of the states is shown in Figure 3.

Using Theorem 3.3 with $W_{d}=0$ and $P_{i}=P, Q_{i}=Q, i=1,2$, it is possible to obtain $K=-[3.1599,3.4971]$ that assures the quadratic stability of the system for $\underline{d}=0$ and $\bar{d}=19$. 


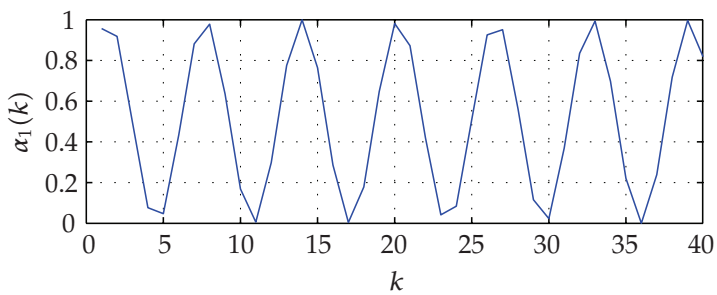

(a)

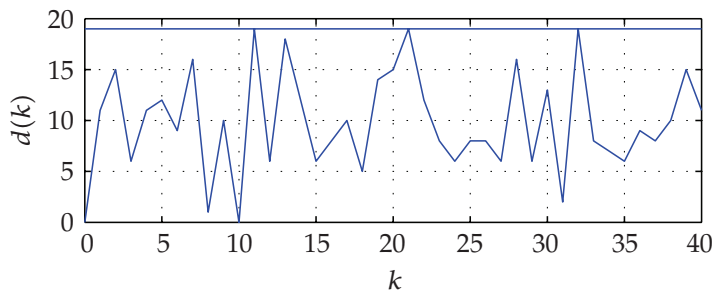

(b)

Figure 5: $\alpha_{1}(k)$ (a) and time-varying delay, $d(k)(\mathrm{b})$.

Therefore, $\tilde{A}(k)=\alpha_{1}(k) \tilde{A}_{1}+\alpha_{2}(k) \tilde{A}_{2}, \widetilde{A}_{d}(k)=\alpha_{1}(k) \tilde{A}_{d 1}+\alpha_{2}(k) \tilde{A}_{d 2}$, and $\widetilde{B}(k)=\alpha_{1}(k) \widetilde{B}_{1}+$ $\alpha_{2}(k) \widetilde{B}_{2}, \alpha(k) \in \Omega$. The closed-loop system behavior is illustrated in Figures 4 and 5 . The state behavior of the closed-loop system is shown in Figure 4(a) and the control signal is presented in the bottom part. This system has been simulated for $\alpha_{1}(k)=0.5(\sin (k)+1)$ and $\alpha_{2}(k)=$ $1-\alpha_{1}(k)$ as seen in Figure 5(a), with initial conditions $x(k)=[1,-1]^{\prime}, k=-19, \ldots, 0$. The time-varying delay $d(k)$ has been randomly generated as indicated in Figure 5(b).

Therefore, this example shows that conditions of Theorem 3.3 can be used in the context of time-varying systems encompassing, in this case, quadratic stability conditions.

\section{Conclusions}

Some sufficient convex conditions were proposed to solve two problems: the robust stability analysis and the synthesis of robust state feedback gains for the class of polytopic discrete-time systems with time-varying delay. The presented LMI conditions include some extra variables and no additional dynamic in the investigated system, thus yielding less conservative results. Some examples, with numerical simulation, are given to demonstrate some relevant characteristics of the proposed design methodology such as robust stabilization using memory or memoryless state feedback gains in the control law, decentralized control, and design for timevarying discrete-time systems with time-varying delay. Some of these examples have been compared with other results available in the literature.

\section{Acknowledgments}

This paper has been supported by the Brazilian agencies CNPq (485496/2006-2) and FAPEMIG (TEC 840/05). The authors are grateful to the anonymous reviewers for their careful reading and valuable suggestions and comments that have helped to improve the presentation of the paper. 


\section{References}

[1] L. Dugard and E. I. Verriest, Stability and Control of Time-Delay Systems, Springer, Berlin, Germany, 1997.

[2] K. Gu, V. L. Kharitonov, and J. Chen, Stability of Time-Delay Systems, Control Engineering, Birkhäuser, Boston, Mass, USA, 2003.

[3] M. S. Mahmoud, Robust Control and Filtering for Time-Delay Systems, vol. 5 of Control Engineering, Marcel Dekker, New York, NY, USA, 2000.

[4] S.-I. Niculescu, Delay Effects on Stability: A Robust Control Approach, vol. 269 of Lecture Notes in Control and Information Sciences, Springer, London, UK, 2001.

[5] S.-I. Niculescu and K. Gu, Eds., Advances in Time-Delay Systems, vol. 38 of Lecture Notes in Computational Science and Engineering, Springer, Berlin, Germany, 2004.

[6] J.-P. Richard, "Time-delay systems: an overview of some recent advances and open problems," Automatica, vol. 39, no. 10, pp. 1667-1694, 2003.

[7] K. J. Åström and B. Wittenmark, Computer Controlled Systems: Theory and Design, Prentice Hall, Englewood Cliffs, NJ, USA, 1984.

[8] F. Gouaisbaut and D. Peaucelle, "A note on the stability of time delay systems," in Proceedings of the 5th IFAC Symposium on Robust Control Design (ROCOND '06), vol. 5, Toulouse, France, July 2006.

[9] P. Shi, E.-K. Boukas, Y. Shi, and R. K. Agarwal, "Optimal guaranteed cost control of uncertain discrete time-delay systems," Journal of Computational and Applied Mathematics, vol. 157, no. 2, pp. 435-451, 2003.

[10] S. Xu, J. Lam, and C. Yang, "Quadratic stability and stabilization of uncertain linear discrete-time systems with state delay," Systems \& Control Letters, vol. 43, no. 2, pp. 77-84, 2001.

[11] S. Xu, "Robust $\mathrm{H}_{\infty}$ filtering for a class of discrete-time uncertain nonlinear systems with state delay," IEEE Transactions on Circuits and Systems I, vol. 49, no. 12, pp. 1853-1859, 2002.

[12] Z. Zuo, J. Wang, and L. Huang, "Robust stabilization for non-linear discrete-time systems," International Journal of Control, vol. 77, no. 4, pp. 384-388, 2004.

[13] G. Hu and E. J. Davison, "Real stability radii of linear time-invariant time-delay systems," Systems $\mathcal{E}$ Control Letters, vol. 50, no. 3, pp. 209-219, 2003.

[14] E. Fridman and U. Shaked, "Stability and guaranteed cost control of uncertain discrete delay systems," International Journal of Control, vol. 78, no. 4, pp. 235-246, 2005.

[15] E. Fridman and U. Shaked, "Delay-dependent $\mathrm{H}_{\infty}$ control of uncertain discrete delay systems," European Journal of Control, vol. 11, no. 1, pp. 29-37, 2005.

[16] E.-K. Boukas and Z. K. Liu, "Robust $\mathrm{H}_{\infty}$ control of discrete-time Markovian jump linear systems with mode-dependent time-delays," IEEE Transactions on Automatic Control, vol. 46, no. 12, pp. 1918-1924, 2001.

[17] W.-H. Chen, Z.-H. Guan, and X. Lu, “Delay-dependent guaranteed cost control for uncertain discretetime systems with delay," IEE Proceedings: Control Theory and Applications, vol. 150, no. 4, pp. 412-416, 2003.

[18] X. G. Liu, R. R. Martin, M. Wu, and M. L. Tang, "Delay-dependent robust stabilisation of discrete-time systems with time-varying delay," IEE Proceedings: Control Theory and Applications, vol. 153, no. 6, pp. 689-702, 2006.

[19] H. Gao, J. Lam, C. Wang, and Y. Wang, "Delay-dependent robust output feedback stabilisation of discrete-time systems with time-varying state delay," IEE Proceedings: Control Theory and Applications, vol. 151, no. 6, pp. 691-698, 2004.

[20] Q.-X. Chen, L. Yu, and W.-A. Zhang, "Delay-dependent output feedback guaranteed cost control for uncertain discrete-time systems with multiple time-varying delays," IEE Proceedings: Control Theory and Applications, vol. 1, no. 1, pp. 97-103, 2007.

[21] E.-K. Boukas, "Discrete-time systems with time-varying time delay: stability and stabilizability," Mathematical Problems in Engineering, vol. 2006, Article ID 42489, 10 pages, 2006.

[22] V. J. S. Leite, S. Tarbouriech, and P. L. D. Peres, "A convex approach for robust state feedback control of discrete-time systems with state delay," in Proceedings of the American Control Conference, pp. 2870 2875, Boston, Mass, USA, June-July 2004.

[23] V. J. S. Leite and P. L. D. Peres, “An improved LMI condition for robust $\boldsymbol{\Phi}$-stability of uncertain polytopic systems," IEEE Transactions on Automatic Control, vol. 48, no. 3, pp. 500-504, 2003. 
[24] R. C. L. F. Oliveira and P. L. D. Peres, "LMI conditions for robust stability analysis based on polynomially parameter-dependent Lyapunov functions," Systems E Control Letters, vol. 55, no. 1, pp. 52-61, 2006.

[25] M. C. de Oliveira and J. C. Geromel, "A class of robust stability conditions where linear parameter dependence of the Lyapunov function is a necessary condition for arbitrary parameter dependence," Systems \& Control Letters, vol. 54, no. 11, pp. 1131-1134, 2005.

[26] P. Gahinet, A. Nemirovskii, A. J. Laub, and M. Chilali, “LMI Control Toolbox User's Guide," The Math Works, Natick, Mass, USA, 1995.

[27] J. F. Sturm, "Using SeDuMi 1.02, a MATLAB toolbox for optimization over symmetric cones," Optimization Methods and Software, vol. 11, no. 1-4, pp. 625-653, 1999. 


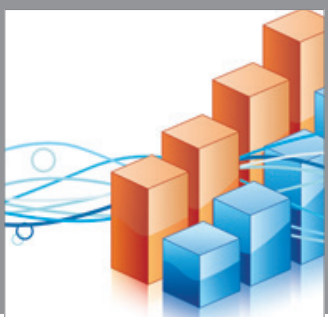

Advances in

Operations Research

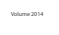

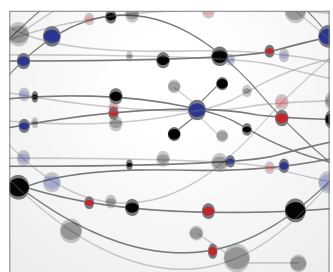

\section{The Scientific} World Journal
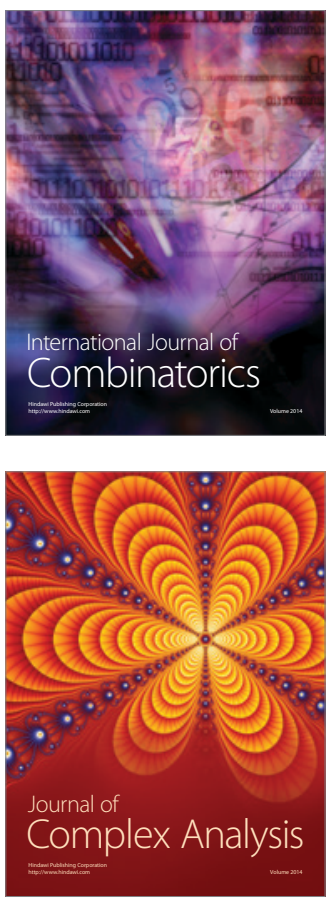

International Journal of

Mathematics and

Mathematical

Sciences
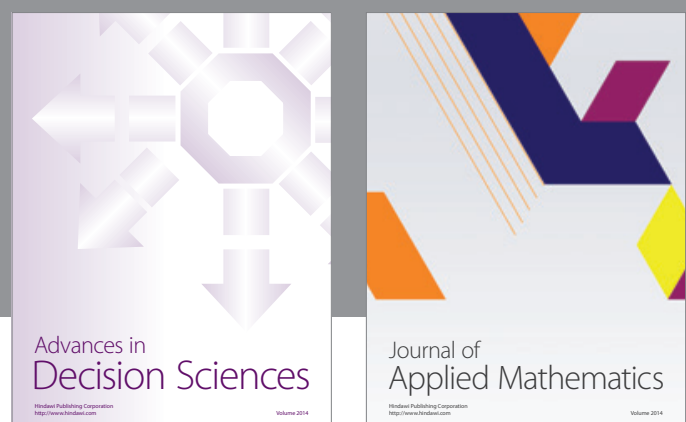

Journal of

Applied Mathematics
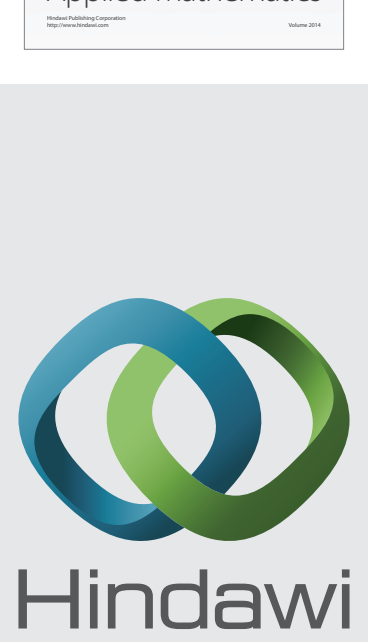

Submit your manuscripts at http://www.hindawi.com
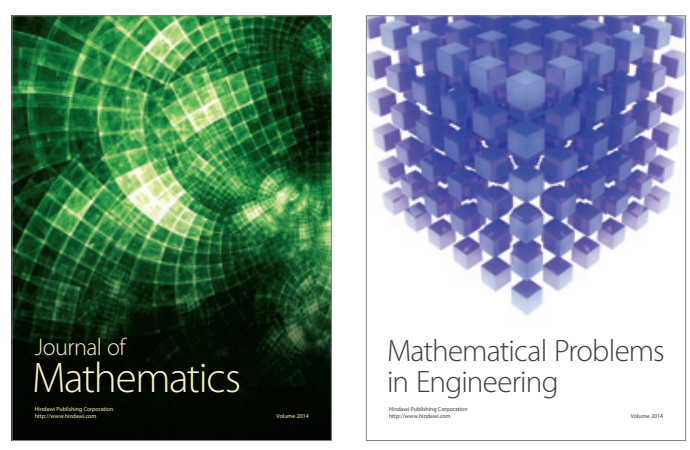

Mathematical Problems in Engineering
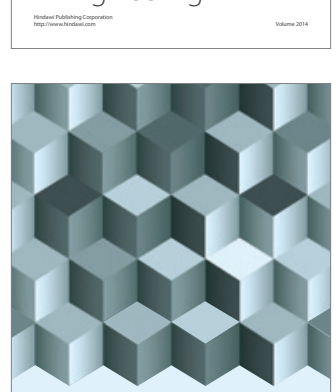

Journal of

Function Spaces
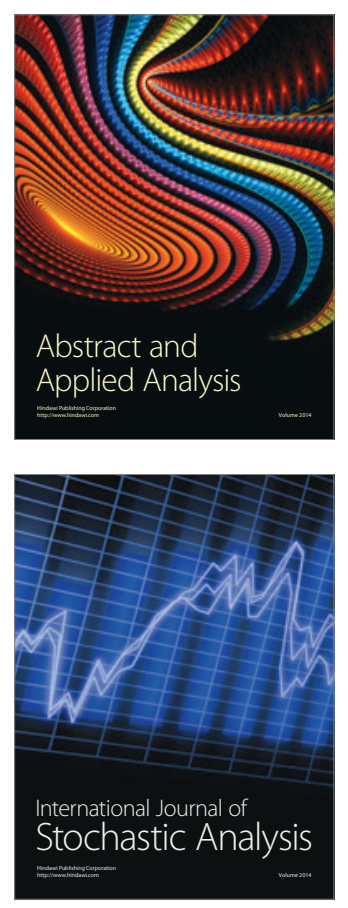

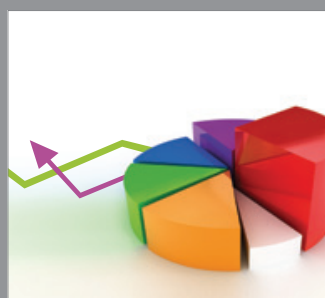

ournal of

Probability and Statistics

Promensencen
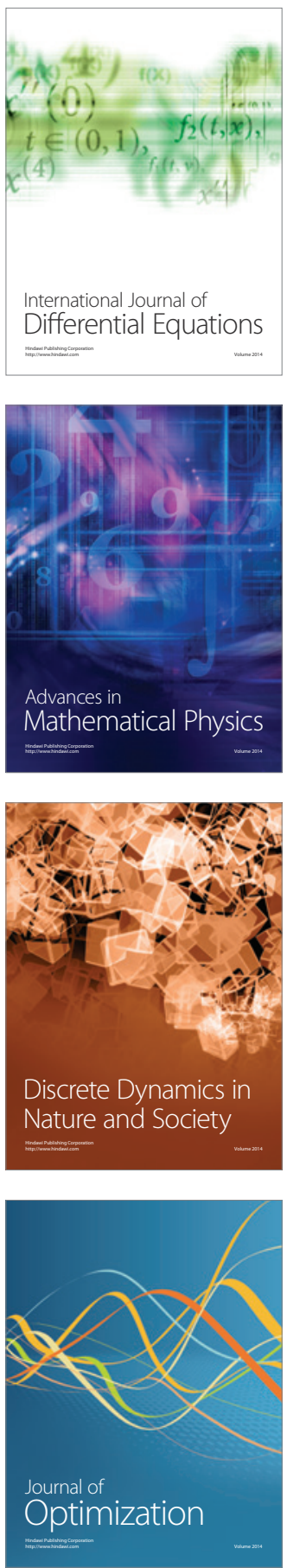